\title{
International Agency for Research on Cancer
}

\section{International Childhood Cancer Day: Much remains to be done to fight childhood cancer}

\begin{abstract}
Lyon, France, 15 February 2016 - New estimates by the International Agency for Research on Cancer (IARC) show that the global occurrence of childhood $^{1}$ cancer is higher than previously assessed. Worldwide, approximately 215000 cancers are diagnosed per year in those younger than 15 years and about 85000 cancers in those aged 15-19 years.
\end{abstract}

These estimates are based on data collected by more than 100 population-based cancer registries in 68 countries around the world in 2001-2010. The full data, which will be published later this year as the third volume of International Incidence of Childhood Cancer (IICC-3), are part of a collaborative project of IARC and the International Association of Cancer Registries (IACR). Supported by the Union for International Cancer Control (UICC), the publication will provide a unique source of information on worldwide childhood cancer incidence.

Cancer is rare in children. In developed countries, it represents less than $1 \%$ of all cancers. But in lowresource settings, where children may make up half of the population, the proportion of childhood cancer can be 5 times higher.

"The proportion of children dying from cancer in poorer countries is unacceptably high, especially when we have the example from richer countries as to what can be achieved through access to care," says $\mathrm{Dr}$ Christopher Wild, IARC Director. "Childhood cancer is a public health problem in developing countries, and more resources should be provided to improve diagnosis, treatment, and infrastructure."

\section{Global burden and incidence pattern}

Compared with the adult population, children are affected by different types of cancer. Almost half of childhood cancers are cancers of blood cells (leukaemia and lymphoma), and the most frequent other malignancies are tumours of the central nervous system and tumours that develop from embryonal tissues. Several tumour types occur almost exclusively in children, such as neuroblastoma, nephroblastoma, and retinoblastoma, whereas carcinomas, which are the predominant type in adults (breast, lung, or stomach cancer), are extremely rare in children.

There are striking differences in the international distribution of childhood cancers. Leukaemia is the most commonly diagnosed cancer in most world regions, where it represents about $35 \%$ of all childhood cancer cases. However, leukaemia is rarely recorded in sub-Saharan Africa. It is not clear whether this is due to missed diagnosis or other reasons.

Certain cancers are very rare in general but do occur with high frequency in some parts of the world. For example, Burkitt lymphoma develops frequently in equatorial African countries, in association with high exposure to infectious agents such as Epstein-Barr virus and malaria. Kaposi sarcoma is the most common childhood cancer in some countries with a high prevalence of HIV infection, such as Uganda. Another notable difference is the very few cases of central nervous system tumours in low-resource

\footnotetext{
${ }^{1}$ Childhood cancer is defined as cancer occurring before 19 years of age.
} 


\section{International Childhood Cancer Day: Much remains to be done to fight childhood cancer}

countries, which could be explained, at least in part, by a lack of appropriate (and expensive) diagnostic facilities for detecting these tumours.

"Childhood cancer is often neglected because cancer is predominantly a disease of ageing populations," says IARC scientist Dr Eva Steliarova-Foucher. "Data from cancer registries are indispensable for bringing to light the full burden of childhood cancer, giving fresh clues to the causes, and for monitoring the impact of efforts to combat the problem."

\section{Child mortality}

Many cancers that affect children can be treated, but cancer remains an important cause of child mortality, with an estimated 80000 cancer-related deaths in children per year worldwide. Although childhood cancer survival rates are about $80 \%$ in high-income countries, they may be as low as $10 \%$ in some countries.

"The lack of diagnosis of many children and adolescents with cancer, as well as the need for improved early detection and rapid referral for treatment, means that childhood cancers that could readily be cured are too often a death sentence," says Professor Tezer Kutluk, a paediatric oncologist from Ankara, Turkey, who is the President of UICC. "It is vital that developing countries get the technical and financial support they need to establish a strong health system that offers to each child the opportunity for cure that we achieve in high-income countries every day."

\section{For more information, please contact}

Véronique Terrasse, Communications Group, at $+33(0) 472738366$ or terrassev@iarc.fr or Dr Nicolas Gaudin, IARC Communications, at com@iarc.fr

The International Agency for Research on Cancer (IARC) is part of the World Health Organization. Its mission is to coordinate and conduct research on the causes of human cancer, the mechanisms of carcinogenesis, and to develop scientific strategies for cancer control. The Agency is involved in both epidemiological and laboratory research and disseminates scientific information through publications, meetings, courses, and fellowships. If you wish your name to be removed from our press release emailing list, please write to com@iarc.fr. 